\title{
MICROTECHNIQUE FOR BLOOD CULTURES
}

BY

\author{
F. RAPPAPORT AND T. GLIKIN \\ From the Municipal Hadassah Hospital, Tel-Aviv, Israel
}

(RECEIVED FOR PUBLICATION, SEPTEMBER 12, 1949)

In the usual bacteriological practice blood for blood cultures is taken from a vein. The incentive for utilizing capillary blood for cultures is based, in part, on a recent concept of the defence mechanism of the capillaries. According to this theory the capillaries filter off the circulating thrombi of bacterial cells and thrombocytes. It is a well-known fact that in subacute bacterial endocarditis the finger-tips are congested with thrombi. There is also reason to believe that in bacteriaemic conditions the concentration of bacteria is higher in the capillary than in the venous blood. Mention, indeed, is made in the literature (Stitt, Clough, and Clough, 1938) of positive typhoid cultures yielded by blood drawn from the finger, simple bile medium being used. In this paper methods for a wider application of the microtechnique are presented.

The cultivation of streptococci and pneumococci as well as of Gram-negative organisms from the capillary blood has been possible. Contamination is avoided by the selective bacteriostatic action of gentian violet as described by Fleming (1942) and Francis (1941) incorporated in the culture media.

\section{Media}

Three media are used.

Medium I.-Dehydrated " difco" brain-heart infusion with P.A.B. and agar, 38 g. ; sodium citrate (tribasic), $2 \mathrm{~g}$., dissolved and made up with distilled water to $1,000 \mathrm{ml}$. The prepared medium is distributed in test-tubes in amounts of 5-7 ml. and sterilized in an autoclave for 30 minutes at $15 \mathrm{lb}$. pressure. To each tube $0.05 \mathrm{ml}$. of sterile " bacto supplement A," containing the necessary amount of gentian violet, is added aseptically. For storage the tubes are wrapped in tinfoil or cellophane.

Medium II.-Kracke blood culture medium. The medium is distributed in quantities of 5-7 ml. in test-tubes and sterilized in an autoclave at $15 \mathrm{lb}$. pressure for 30 minutes. To each tube $0.1 \mathrm{ml}$. of " bacto supplement $A$ " is added aseptically, and the test-tubes are wrapped as described above. Better results are obtained when the dehydrated medium is dissolved in fresh-water fish broth-e.g., using Barbiceps longus (Rappaport and Glikin, 1943) instead of water.

Medium III.-3\% glucose bile medium. With this medium heavy precipitation after incubation indicates the presence of glucose fermenters (Rappaport, 1947).

\section{Method}

Blood is best drawn from the finger-tip or, in infants, from the heel. The site is disinfected by means of a pad of absorbent cellulose. The pads are made of rectangular pieces of absorbent cellulose $(11 \times 8 \mathrm{~cm}$.) stapled to larger squares of parchment paper 
with a margin all round of $1 \mathrm{~cm}$. To sterilize, the pads are folded in the middle with the cellulose inside, placed in envelopes, and autoclaved. Before use the pad is removed from the envelope, opened, and the cellulose wetted with "septoform" (i.e., glycerin, $10 \mathrm{~g}$., $40 \%$ formalin, $8 \mathrm{ml}$., sap. mol., $6 \mathrm{~g}$., or spiritus saponatus, $30 \mathrm{ml}$., made up with alcohol to $1,000 \mathrm{ml}$., mixed and filtered). The pad is then wrapped round the fingertip and kept in place by a rubber band. The disinfectant is allowed to act for three minutes, whereupon the finger is wiped dry with a second sterile pad. A superficial puncture is made with a lancet, flamed just before use, and a few drops of blood extracted. The blood is taken up either in a 1-ml. sterile syringe (the plunger lubricated with paraffin oil before sterilization) or in a small sterile pipette (Fig. 1). A rubber tube is connected to the upper end of the pipette, which is still held in the inner test-tube, and just before use the pipette is taken out of this tube. While blood is being drawn from the finger-tip, care must be taken that no air enters the pipette, as it may be a source of contamination. Each of the three media is aseptically inoculated with the blood and incubated from 12 to 16 hours for pneumococci, 24 to 48 hours for other organisms, and six to seven days in an atmosphere of $10 \%$ $\mathrm{CO}_{2}$ for Brucella. One of the two smears made from the subcultures is stained by the Gram method and the other with methylene blue, both after alcohol fixation. Methylene blue is used because Gram-negative bacteria grown under these conditions take on the fuchsin counterstain with difficulty. In methyleneblue-stained smears the microorganisms of the enteric group appear as bipolar rods closely resembling Pasteurella.

\section{Discussion}

The results obtained by this technique compared with those using venous blood have so far been satisfactory. Moreover, in some cases of subacute bacterial endocarditis in which venous blood cultures were negative the micro-method enabled streptococci to be cultured. Positive capillary-blood cultures, usually free from contaminants, were obtained in cases of typhoid fever
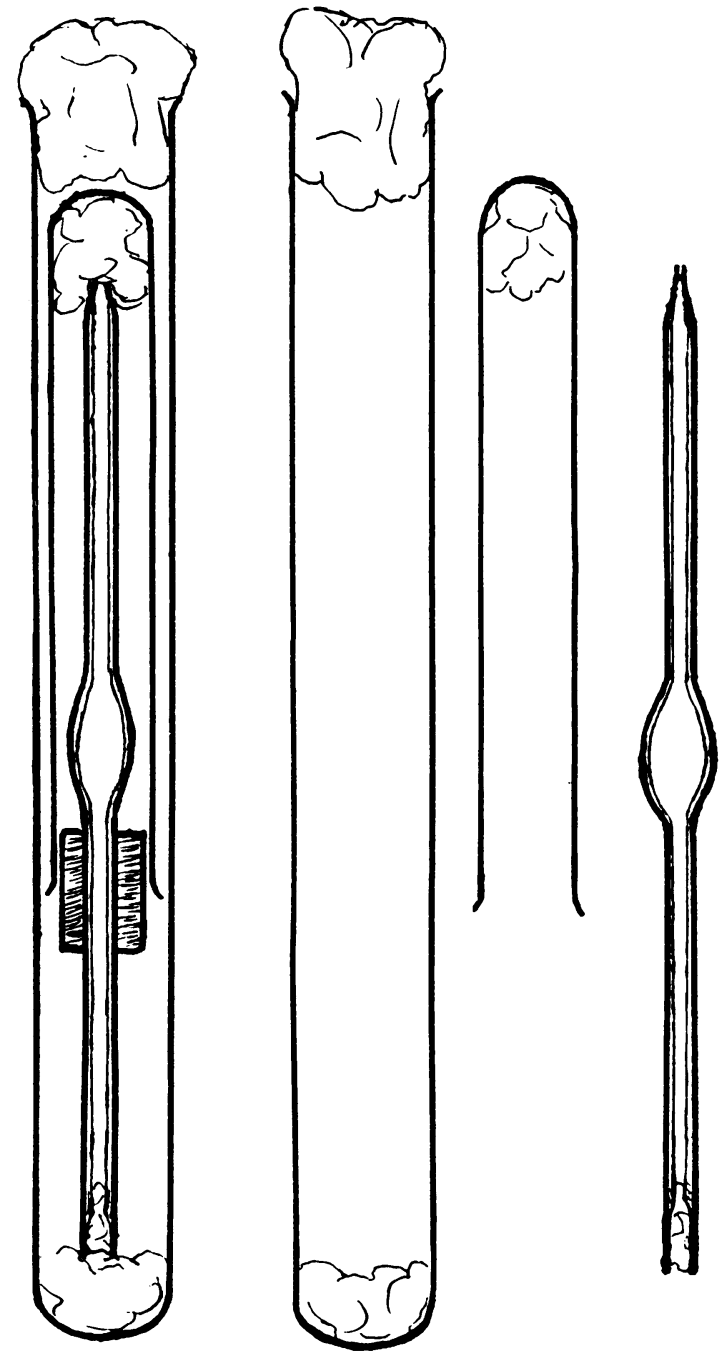

FIG. 1.-Sterile pipette for use in the microtechnique for blood cultures. 
(Salm. typhi), paratyphoid (Salm. para typhi A, B, and C), subacute bacterial endocarditis (Str. viridans), scarlet fever (Str. pyogenes), pneumonia (Str. pneumoniae), and septicaemia (anaerobic streptococci). Brucella melitensis has also been isolated by this method.

In our experience one negative result should not be considered as final. If a septic condition is suspected most reliable results are obtained when cultures are repeated at daily intervals.

The technique we have described is much more convenient than venipuncture, particularly in cases where sufficiently prominent veins are difficult to find. The method is also of special importance when repeated blood culture from the same patient is required. At the very least, this method should prove to be a valuable auxiliary to the usual method.

Fleming, A. (1942). Brit. med. J., 1, 547.

\section{REFERENCES}

Francis, A. E. (1941). Lancet, 2, 159.

Rappaport, F. (1947). Amer. J.clin. Path., 17, 904.

and Glikin, T. (1943). Acta med. orient., Tel-Aviv, 2, 148.

Stitt, E. R., Clough, P. W., and Clough, M. C. (1938). Practical Bacteriology, Hematology, and Parasitology. H. N. Lewis \& Co. 